EPJ Web of Conferences 71, 00046 (2014)

DOI: 10.1051/epjconf/20147100046

(C) Owned by the authors, published by EDP Sciences, 2014

\title{
Overview of the nucleon spin studies at COMPASS
}

\author{
Celso Franco, on behalf of the COMPASS collaboration ${ }^{1, a}$ \\ ${ }^{1}$ LIP, Av. Elias Garcia 14 - 10 1000-149 Lisboa
}

\begin{abstract}
The COMPASS experiment [1] at CERN is one of the leading experiments studying the spin structure of the nucleon. These studies are being carried on since 2002, by measuring hadrons produced in deep inelastic scattering (DIS) of $160 \mathrm{GeV} / \mathrm{c}$ polarised muons off different polarised targets $\left(\mathrm{NH}_{3}\right.$ for polarised protons and ${ }^{6} \mathrm{LiD}$ for polarised deuterons). One of the main goals is to determine how the total longitudinal spin projection of the nucleon, $1 / 2$, is distributed among its constituents, quarks and gluons. We review here the recent results on the quark and gluon helicities obtained by COMPASS, using a longitudinally polarised target. However, the understanding of the nucleon (spin) structure based only on the parton helicities is not in any way complete. It basically provides us with a one-dimensional picture in a longitudinal momentum space. Therefore, COMPASS also studies the transverse momentum dependent parton distributions (TMDs) with a transversely polarised target. Concerning the TMDs, the latest results on the Collins and Sivers asymmetries will be shown. The former is sensitive to the transverse spin structure of the nucleon, while the latter reflects the correlations between the quarks transverse momentum and the nucleon spin.
\end{abstract}

\section{Introduction}

In perturbative QCD (pQCD), the leading twist contribution to a parton distribution function can be written in collinear approximation as

$$
\Phi(x)_{\text {coll }}^{T w-2}=\frac{1}{2}\left[q(x)+\gamma_{5} \Delta q(x) S_{L}+\gamma_{5} \Delta_{T} q(x) S_{T} \gamma_{1}\right]
$$

The quantity $q(x)$ describes the unpolarised parton distribution as a function of the longitudinal momentum fraction carried by the parton, $\Delta q(x)\left(\Delta_{T} q(x)\right)$ represents the longitudinally (transversely) polarised parton distribution function and $S_{L}\left(S_{T}\right)$ stands for the longitudinal (transverse) spin of the nucleon. In COMPASS, the polarised PDFs are investigated via DIS processes for all the light quarks $(u, d$ and $s)$ and also for gluons. The latter is possible by studying the next-to-leading order (NLO) interaction of photon-gluon fusion (PGF).

One of the major goals of COMPASS is to understand how the longitudinal spin projection of the nucleon, $1 / 2$, is built up from its basic constituents:

$$
\frac{S_{z}^{N}}{\hbar}=\frac{1}{2}=\frac{1}{2} \Delta \Sigma\left(=\sum_{q} \Delta q\right)+L_{z}^{q}+\Delta G+L_{z}^{g}
$$

\footnotetext{
ae-mail: celso@lip.pt
} 
$\Delta \Sigma(\Delta G)$ represents the total quark (gluon) helicity and $L_{z}^{q}\left(L_{z}^{g}\right)$ represents the orbital angular momentum (OAM) of quarks (gluons). The most recent results on the quark and gluon helicities are reviewed here. Measurements of transverse spin asymmetries leading to the determination of the transverse spin distributions of quarks and to the Sivers function are also discussed. The latter is sensitive to the OAM of quarks and, therefore, may be of crucial importance for the determination of one of the remaining unknowns in Eq. 2. This article will conclude with a summary of the future plans concerning the study of OAM, TMDs and GPDs (generalised parton distributions).

\section{Longitudinal Spin Structure of the Nucleon}

By using the world data on the longitudinal spin structure functions $g_{1}^{d}\left(x, Q^{2}\right)$ and $g_{1}^{p}\left(x, Q^{2}\right)$ (cf. Fig. 1), the total contribution of quarks to the nucleon spin can be obtained [2]. Nowadays the total quark helicity is confirmed to be $30 \%: \Delta \Sigma=0.30 \pm 0.01 \pm 0.02$. However, the precise determination of the quark helicity per flavour and of the gluon helicity is still ongoing. These 2 topics are discussed in the next subsections.
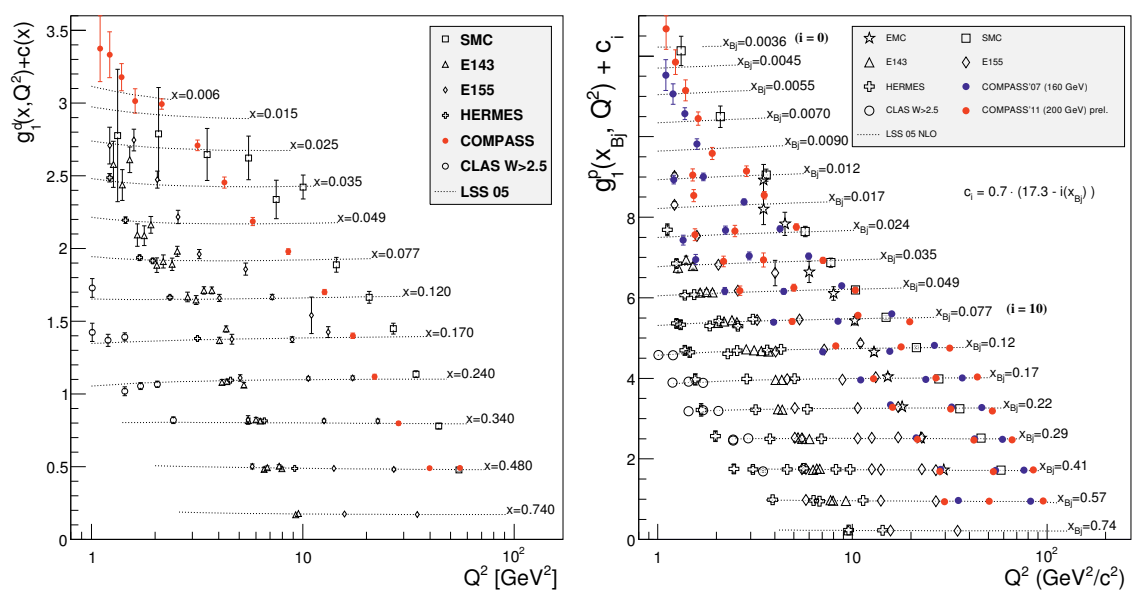

Figure 1. World data on $g_{1}^{d}$ (left) and $g_{1}^{p}$ (right). The COMPASS improvements in precision and in kinematic coverage are evident.

\subsection{Quark Helicities}

COMPASS measures semi-inclusive spin asymmetries of kaons and pions, on both proton and deuteron targets, in order to study the quark helicities. In the LO approximation, this hadronic asymmetry is defined by

$$
A_{1}^{h}=\frac{\sum_{q} e_{q}^{2} \Delta q\left(x, Q^{2}\right) D_{q}^{h}\left(z, Q^{2}\right)}{\sum_{q} e_{q}^{2} q\left(x, Q^{2}\right) D_{q}^{h}\left(z, Q^{2}\right)}
$$

where $e_{q}$ is the electric charge of a given quark flavour, $\Delta q$ (q) the polarised (unpolarised) parton distribution function and $D_{q}^{h}$ the fragmentation function (FF) of the struck quark to a hadron $h$. A total of 8 asymmetries are measured via SIDIS (semi-inclusive DIS) processes at COMPASS, namely $A_{1}^{\pi^{+}}$, $A_{1}^{\pi^{-}}, A_{1}^{K^{+}}$and $A_{1}^{K^{-}}$for each of the considered targets. By taking also into account the 2 inclusive spin 
asymmetries, which were used for the extraction of $g_{1}^{d}$ and $g_{1}^{p}$, one is able to determine the following quark helicities [3]: $\Delta u, \Delta \bar{u}, \Delta d, \Delta \bar{d}$ and $\Delta s$. The results were obtained using the DSS parametrisation for $D_{q}$ and the MRST04 parametrisation for $q$ and are shown in Fig.2:

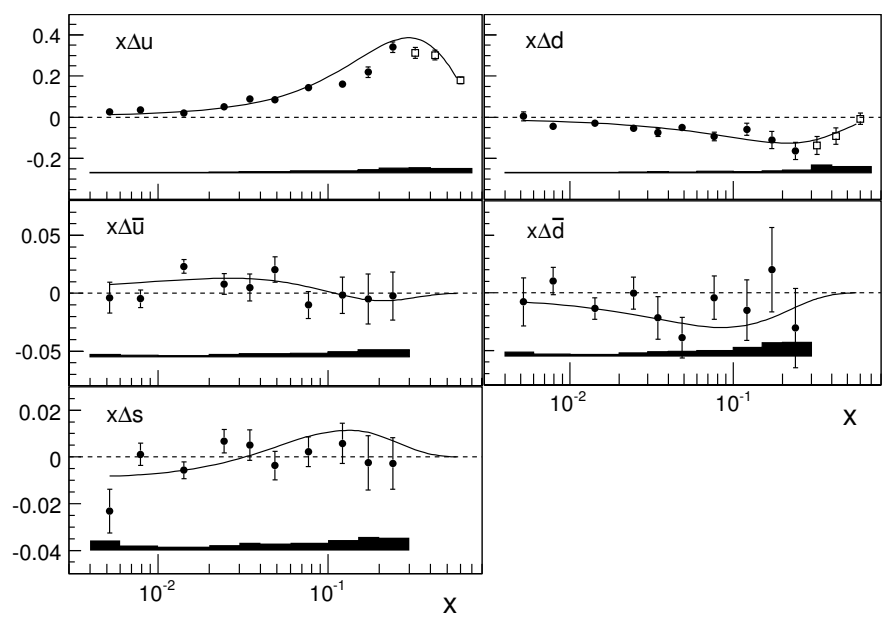

Figure 2. COMPASS results on the light quark helicities under the assumption of $\Delta s=\Delta \bar{s}$. The curves correspond to a global NLO fit obtained from DSSV[4].

It is known from the inclusive asymmetries that the strange quark helicity is negative: $\Delta s(x)+\Delta \bar{s}(x)=$ $-0.08 \pm 0.01 \pm 0.02$ [2]. However, from Fig. 2 one obtains $\Delta s=-0.01 \pm 0.01 \pm 0.01$. Since the semi-inclusive result is strongly dependent on the choice of the FFs used (cf Eq. 3), COMPASS is measuring hadron multiplicities in order to better constrain the needed FFs. At LO, the number of hadrons per DIS event (hadron multiplicities) is defined by

$$
\frac{d M^{h}}{d z}=\frac{\sum_{q} e_{q}^{2} q\left(x, Q^{2}\right) D_{q}^{h}\left(z, Q^{2}\right)}{\sum_{q} e_{q}^{2} q\left(x, Q^{2}\right)}
$$

The latest results on the deuteron data for charged $K$ multiplicities are shown in Fig. $3\left(\pi^{+}\right.$and $\pi^{-}$ multiplicities are also available). This data can be used in global analyses to improve our knowledge on FFs, $\Delta s(x)$ and $s(x)$.

\subsection{Gluon Polarisation}

There are two ways to study the gluon polarisation $\Delta g / g$ in DIS: an indirect method using NLOpQCD analyses and a more direct one using the PGF process. The former uses QCD fits to the $g_{1}^{p}$ and $g_{1}^{d}$ data for the extraction of the gluon helicity $\Delta G=\int_{0}^{1} \Delta g(x) d x$ (via DGLAP evolution). The main drawback, however, is the existence of a large uncertainty caused by the limited kinematic coverage of the measurements (cf. Fig. 1). Consequently, the determination of $\Delta G$ from QCD fits must be complemented by direct measurements. In COMPASS two main analyses were followed: open charm and high $p_{T}$ hadron pairs. The first method is free from physical background at LO. This 


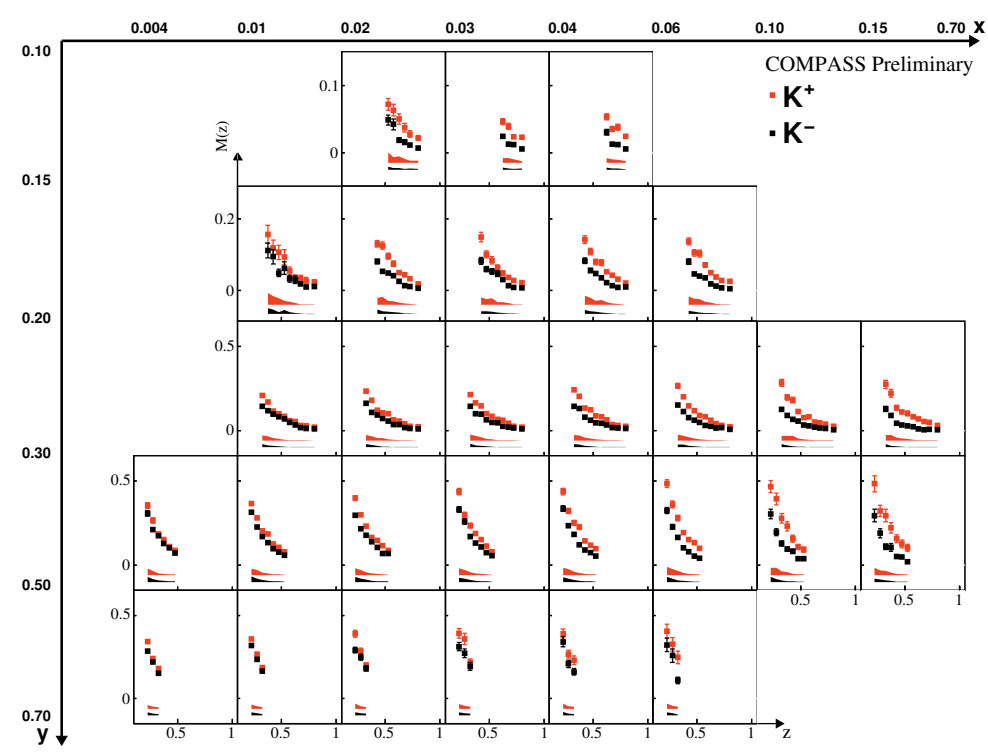

Figure 3. $K^{+}$and $K^{-}$multiplicities in kinematic bins (deuteron data only).

fact is justified by a negligible quantity of intrinsic-charm in the nucleon in the COMPASS kinematic domain $\left(x_{B j}<0.1\right)[5,6]$. The open charm is tagged by the invariant mass reconstruction of $D^{0}$ mesons, via detection of their decay products. Since these mesons can only result from interactions with a gluon inside the polarised nucleon (true at LO and with small exceptions at NLO), the extraction of $\Delta g / g$ can be performed using the following $D^{0}$ asymmetries (at an average $Q^{2}$ ):

$$
A^{D^{0}}=f P_{t} P_{\mu}\left\langle\hat{a}_{L L}\right\rangle\left(\frac{\Delta g}{g}\langle x\rangle\right) \quad \text { with } \quad \hat{a}_{L L} \equiv \frac{\Delta \hat{\sigma}_{\mu g}}{\hat{\sigma}_{\mu g}}=\left(\frac{\hat{\sigma}_{\mu g}^{\leftrightarrows}-\hat{\sigma}_{\mu g}^{\overleftarrow{\leftrightarrows}}}{\hat{\sigma}_{\mu g}^{\leftrightarrows}+\hat{\sigma}_{\mu g}^{\overleftarrow{\leftrightarrows}}}\right)
$$

where $f, P_{t}$ and $P_{\mu}$ are the fraction of polarisable material inside the target, the target polarisation and the beam polarisation respectively. The partonic asymmetry $\hat{a}_{L L}$ is obtained from a Monte Carlo simulation of the PGF process at COMPASS and, thereafter, it is parametrised by a Neural Network in order to allow us to use $\hat{a}_{L L}$ on data. In order to overcome the limited charm statistics at COMPASS, a weighted method was developed to minimise the statistical error of $\Delta g / g$ [7]. Since this method implies an event-by-event analysis, a Neural Network is used to distinguish the PGF events from the combinatorial background in the $D^{0}$ mass spectra. The open charm analysis is performed at $\mathrm{LO}$ and also at NLO in QCD. Both results are shown in Fig. 4 (for details see [7]).

In the second method hadron pairs with high transverse momenta $p_{T}$ are selected. In this case there are several contributions to the measured asymmetries: LO processes, QCD Compton and PGF processes. For low $Q^{2}$ events there are also resolved photon contributions. The analysis is done independently for data with $Q^{2}>1(\mathrm{GeV} / \mathrm{c})^{2}$ and $Q^{2}<1(\mathrm{GeV} / \mathrm{c})^{2}$. Despite the large statistics available, this method has to face a more complicated analysis: in order to extract $\Delta g / g$ from the high- $p_{T}$ asymmetries we need to add to Eq. 5 as many terms as the existing physical processes (with $\Delta g / g$ replaced by $A_{1}^{L O}$ and $A_{1}^{\text {Compton }}$ ). The partonic asymmetries, as well as the fractions corresponding to each phys- 
ical process, are also determined from a Monte Carlo simulation of the COMPASS experiment [8]. The high- $p_{T}$ results were only obtained at LO and are shown in Fig. 4 (left). They agree with the open charm point from COMPASS and with the high- $p_{T}$ points from SMC and HERMES. The most fair conclusion than one can draw from such plot is that $\Delta g / g$ is small and compatible with zero within $x_{g} \in[0.07,0.2]$.
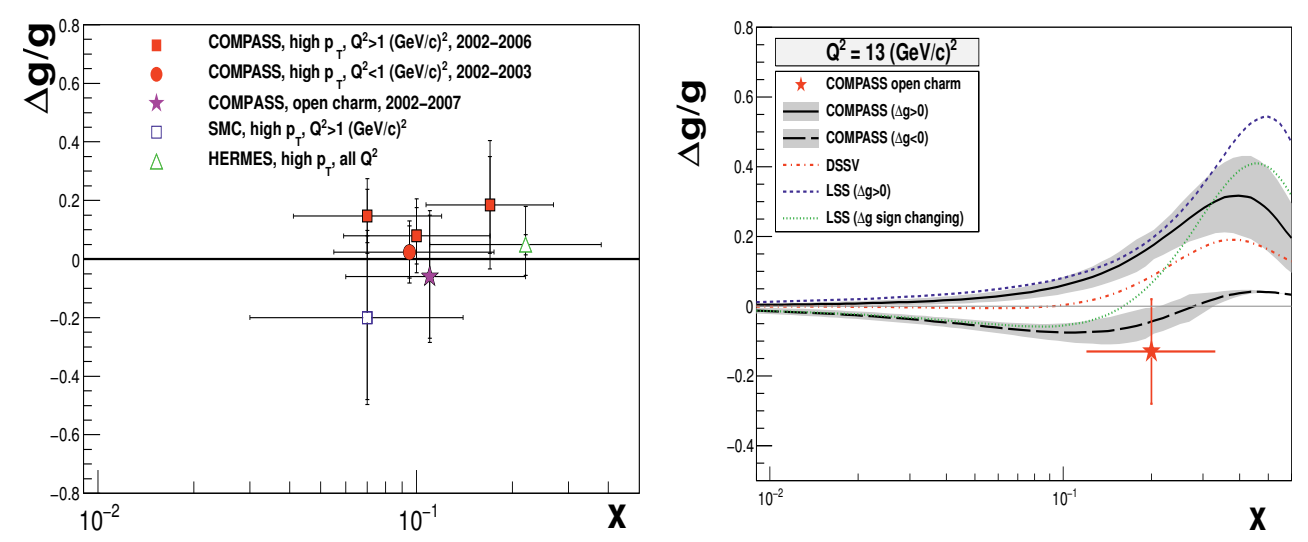

Figure 4. COMPASS results of $\Delta g / g$ obtained at LO (left) and at NLO (right) in QCD. The NLO result can be compared with the existing QCD fits. The COMPASS fits are constrained by the experimental measurement.

\section{Transversity and TMD}

Besides the longitudinally polarised structure function $g_{1}\left(x, Q^{2}\right)$ and the unpolarised structure function $F_{1}\left(x, Q^{2}\right)$, the Transversity function $h_{1}\left(x, Q^{2}\right)$ is the remaining necessary ingredient to describe the nucleon structure at leading twist. The function $h_{1}\left(x, Q^{2}\right)$ is linked to the distribution of transversely polarised partons and can be accessed in SIDIS when using a transversely polarised target. This is possible through a measurement of the so called Collins asymmetry, which is sensitive to the correlation between the outgoing hadron direction (in SIDIS) and the initial quark transverse spin. Therefore, the Collins asymmetry can be used to determine the quark transverse spin distributions $\Delta_{T} u(x)$ and $\Delta_{T} d(x)$. This asymmetry has a $\sin \left(\phi_{h}+\phi_{S}\right)$ modulation, where $\phi_{h}$ represents the azimuthal angle of the outgoing hadron and $\phi_{S}$ the azimuthal angle of the nucleon spin. It is measured simultaneously with other azimuthal asymmetries by fitting the various angular modulations of the outgoing hadron. The Collins asymmetry is defined by

$$
A_{\text {Coll }}=\frac{\sum_{q} e_{q}^{2} h_{1}^{q}\left(x, Q^{2}\right) \otimes H_{1 q}^{\perp h}\left(z,, Q^{2}, p_{T}\right)}{\sum_{q} e_{q}^{2} F_{1}^{q}\left(x, Q^{2}\right) \otimes D_{1 q}^{h}\left(z, Q^{2}\right)}
$$

Here, $F_{1}^{q}$ represents the unpolarised structure function, $D_{1 q}^{h}$ the quark fragmentation function and $H_{1 q}^{\perp h}$ is the so called Collins fragmentation function (depending on spin). The latest results from COMPASS on $A_{\text {Coll }}$ can be seen in Fig. 5. One can confirm the existence of a large signal in the proton data. The zero compatible asymmetry which is seen in the deuteron data is understood as a cancellation between the $u$ and $d$ quarks in the deuteron molecule. More details can be found in [9].

If one takes into account the intrinsic transverse momentum of partons, $k_{T}, 5$ additional TMDs are needed to describe the nucleon structure at leading twist. In particular, the Sivers TMD $f_{1 T}^{\perp}\left(x, k_{T}\right)$ is 

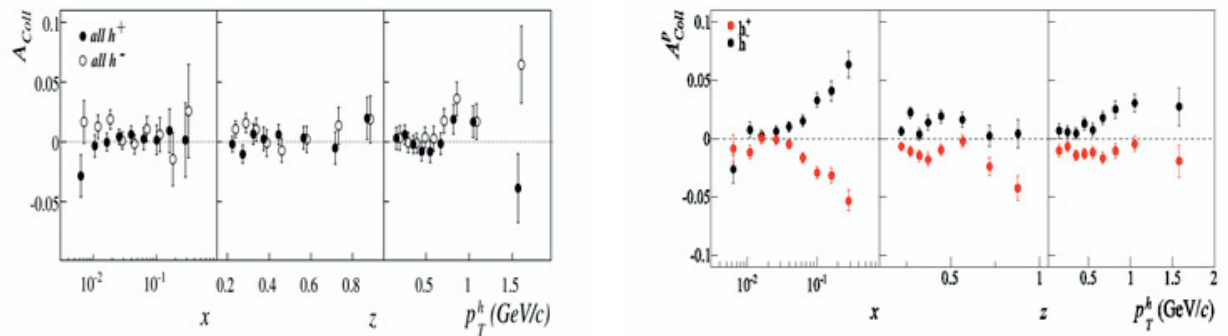

Figure 5. COMPASS results on the Collins asymmetry for deuterons (left) and protons (right).

of extreme importance. Since it correlates the nucleon transverse spin with the quarks $k_{T}$, this TMD is quite sensitive to the quarks OAM (one of the unknowns in Eq. 2). The Sivers function can be determined through a global analysis of the experimental Sivers asymmetry:

$$
A_{\text {Siv }}=\frac{\sum_{q} e_{q}^{2} f_{1 T}^{\perp q}\left(x, Q^{2}, k_{T}\right) \otimes D_{1 q}^{h}\left(z, Q^{2}\right)}{\sum_{q} e_{q}^{2} F_{1}^{q}\left(x, Q^{2}\right) \otimes D_{1 q}^{h}\left(z, Q^{2}\right)}
$$

$A_{S i v}$ is measured at COMPASS by fitting its specific $\sin \left(\phi_{h}-\phi_{S}\right)$ modulation. The results are shown in Fig. 6. This time a large signal is seen only for the positive hadrons of the proton data. For more details see [10]
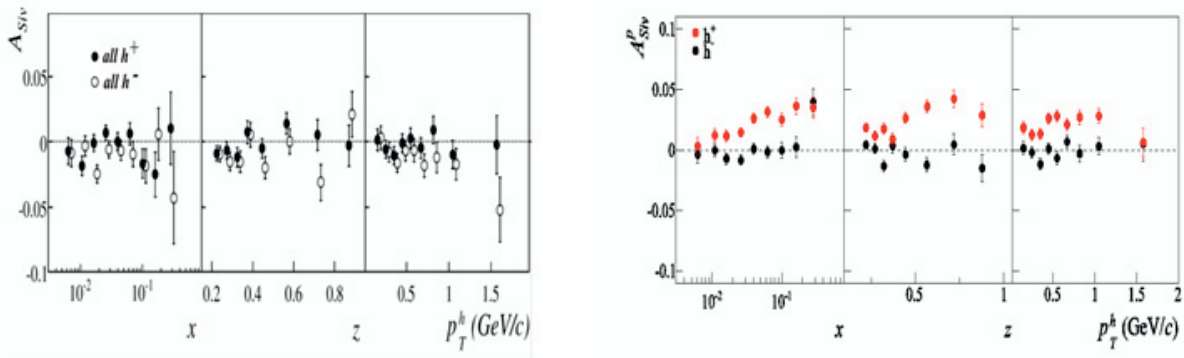

Figure 6. COMPASS results on the Sivers asymmetry for deuterons (left) and protons (right)

Preliminary results on the transversity PDF and on the Sivers TMD for the $u$ and $d$ quarks, using the COMPASS and HERMES asymmetries $A_{\text {Coll }}$ and $A_{\text {Siv }}$, are shown in [11].

\section{Outlook}

From 2014 onwards two new topics will be investigated at COMPASS [12]. One of them is the study of 4 TMDs (Sivers, Boer-Mulders, Transversity and Pretzelosity) with the first ever polarised Drell- 
Yan experiment. The experiment will consist on a $190 \mathrm{GeV} / \mathrm{c} \pi^{-}$beam scattering off a transversely polarised proton target $\left(\mathrm{NH}_{3}\right)$, and the analysis will be focused on the dimuon channel to take advantage of the excellent muon detection by the COMPASS spectrometer [1]. Besides the study of the TMDs distribution, the major goal is to verify the expected sign change of the Sivers and BoerMulders TMDs when accessed via SIDIS or Drell-Yan. The observation of the above mentioned sign change is crucial for our current understanding of TMDs and, by performing both measurements with the same spectrometer, COMPASS has privileged conditions to test this important QCD prediction. As a bonus the Drell-Yan experiment will also allow us to study the production mechanism and polarisation of the $J / \Psi$. These two goals can be achieved with the already approved one year of data taking in 2015. With a second year of data taking, possibly in 2018, it will be possible to study the TMDs as a function of $x_{F}$ and $p_{T}$.

The other major topic will be investigated after the data taking of 2016 and 2017. The goal is the study of GPDs through the deeply virtual compton scattering (DVCS) of $\mu^{+}$and $\mu^{-}$off a liquid hydrogen target. They will be determined from the analysis of the azimuthal dependence of the DVCS cross-section. Since the GPDs correlate the transverse spatial and the longitudinal momentum degrees of freedom, their knowledge will provide us the needed ingredients to obtain tomographic images of the proton. Besides this major breakthrough in the understanding of the nucleon structure, the GPDs are also the ideal tool to access the OAM of quarks via the Ji sum rule:

$$
J^{q}=\frac{1}{2} \Delta \Sigma+L^{q}=\frac{1}{2} \int_{0}^{1} d x x\left[H^{q}(x, \xi, t)+E^{q}(x, \xi, t)\right]
$$

where $H^{q}$ and $E^{q}$ represent two of the four GPDs that will be measured at COMPASS.

\section{Summary}

The total quark contribution to the nucleon spin was measured with high precision and amounts only to $30 \%$. The extraction of quark helicities from SIDIS data was performed for all light flavours. However, more knowledge on fragmentation functions is required for a more reliable extraction of $\Delta s(x)$. In order to better constrain the needed FFs, preliminary multiplicities of charged kaons and pions were already made available by COMPASS.

The gluon contribution to the nucleon spin was found to be small and compatible with zero in the $x_{g}$ region of the measurements. More polarised data on $g_{1}\left(x, Q^{2}\right)$ is needed to reduce the $\Delta G$ uncertainty resulting from the NLO-pQCD analyses.

Precise results on the Collins and Sivers asymmetries were shown. By being non-zero, the latter points to a relevant contribution of the quarks OAM to the nucleon spin (and also to the importance of $k_{T}$ in the description of the nucleon structure).

An exciting physics program is in preparation for the near future. The expected outcome will be a kind of 3D imaging of the nucleon, both in the momentum space (TMDs) and in the position space (GPDs). A decisive step towards a proper understanding of the nucleon spin will also be given by determining the quarks OAM via the Ji sum rule.

\section{References}

[1] COMPASS, P. Abbon et al., The COMPASS experiment at CERN, Nucl. Inst. Meth. A577, 455 (2007).

[2] COMPASS, V. Yu Alexakhin et al., Spin asymmetry $A_{1}^{d}$ and the spin-dependent structure function $g_{1}^{d}$ of the deuteron at low values of $x$ and $Q^{2}$, Phys. Lett. B 647 (2007) 8. 
[3] COMPASS, M. G. Alekseev et al., Flavour Separation of Helicity Distributions from Deep Inelastic Muon-Deuteron Scattering, Phys. Lett. B 680 (2009) 217.

[4] D. de Florian, R. Sassot, M. Stratmann and W. Vogelsang (DSSV), Phys. Rev. Lett. 101 (2008).

[5] Johan Alwall, Quark Asymmetries and Intrinsic Charm in Nucleons, Hep-ph/0508126.

[6] S. J. Brodsky, P. Hoyer, C. Peterson and N. Sakai, The intrinsic charm of the proton, Phys. Lett. B93 (1980) 451.

[7] COMPASS, M. G. Alekseev et al., Leading and Next-to-Leading Order Gluon Polarisation in the Nucleon and Longitudinal Double Spin Asymmetries from Open Charm Muoproduction, Phys. Rev. D 87 (2013) 052018.

[8] COMPASS, M. G. Alekseev et al. et al, Leading order determination of the gluon polarisation from DIS events with high-pT hadron pairs, Phys. Lett. B 718 (2013) 922.

[9] COMPASS, C. Adolph et al. et al, Experimental investigation of transverse spin asymmetries in muon-p SIDIS processes: Collins asymmetries, Phys. Lett. B 717 (2012) 376.

[10] COMPASS, C. Adolph et al. et al, Experimental investigation of transverse spin asymmetries in muon-p SIDIS processes: Sivers asymmetries, Phys. Lett. B 717 (2012) 383.

[11] Slides: https: //indico. cern. ch/getFile.py/access? contribId=393\&sessionId=104\&resID= Q\&materialld=slides\&confId $=198153$

[12] COMPASS Collaboration, COMPASS-II proposal, CERN-SPSC.2010-014, SPSC-P-340, 17 May 2010. 